\title{
Modeling leaderless transcription and atypical genes results in more accurate gene prediction in prokaryotes
}

\author{
Alexandre Lomsadze, ${ }^{1,2,6}$ Karl Gemayel, ${ }^{3,6}$ Shiyuyun Tang, ${ }^{4}$ \\ and Mark Borodovsky ${ }^{1,2,3,4,5}$ \\ ${ }^{1}$ Wallace H. Coulter Department of Biomedical Engineering, Georgia Tech, Atlanta, Georgia 30332, USA; ${ }^{2}$ Gene Probe, Incorporated, \\ Atlanta, Georgia 30324, USA; ${ }^{3}$ School of Computational Science and Engineering, Georgia Tech, Atlanta, Georgia 30332, USA; \\ ${ }^{4}$ School of Biological Sciences, Georgia Tech, Atlanta, Georgia 30332, USA; ${ }^{5}$ Department of Biological and Medical Physics, \\ Moscow Institute of Physics and Technology, Moscow, 141700, Russia
}

In a conventional view of the prokaryotic genome organization, promoters precede operons and ribosome binding sites (RBSs) with Shine-Dalgarno consensus precede genes. However, recent experimental research suggesting a more diverse view motivated us to develop an algorithm with improved gene-finding accuracy. We describe GeneMarkS-2, an ab initio algorithm that uses a model derived by self-training for finding species-specific (native) genes, along with an array of precomputed "heuristic" models designed to identify harder-to-detect genes (likely horizontally transferred). Importantly, we designed GeneMarkS-2 to identify several types of distinct sequence patterns (signals) involved in gene expression control, among them the patterns characteristic for leaderless transcription as well as noncanonical RBS patterns. To assess the accuracy of GeneMarkS-2, we used genes validated by COG (Clusters of Orthologous Groups) annotation, proteomics experiments, and $\mathrm{N}$-terminal protein sequencing. We observed that GeneMarkS-2 performed better on average in all accuracy measures when compared with the current state-of-the-art gene prediction tools. Furthermore, the screening of $\sim 5000$ representative prokaryotic genomes made by GeneMarkS-2 predicted frequent leaderless transcription in both archaea and bacteria. We also observed that the RBS sites in some species with leadered transcription did not necessarily exhibit the Shine-Dalgarno consensus. The modeling of different types of sequence motifs regulating gene expression prompted a division of prokaryotic genomes into five categories with distinct sequence patterns around the gene starts.

[Supplemental material is available for this article.]

Since the number of microbial species on Earth is estimated to be larger than $10^{12}$ (Locey and Lennon 2016), the exponential growth of the number of sequenced prokaryotic genomes, currently $\sim 10^{5}$, is likely to continue for quite a while. Along this path, we will continue to see genomes with large numbers of genes not detectable by mapping protein orthologs. Thus, improving the accuracy of $a b$ initio gene prediction remains an important task.

The proliferation of RNA-seq presented an opportunity for more accurate inference of exon-intron structures of eukaryotic genes. Transcriptomes of prokaryotes, however, were thought to be less important for gene finding since the accuracy of ab initio prediction of a whole gene (being uninterrupted ORF) is significantly higher. Nevertheless, recent innovations in the NGS techniques led to generating new kinds of data whose impact has yet to be fully appreciated.

For example, dRNA-seq, the differential RNA sequencing technique (Sharma et al. 2010; Sharma and Vogel 2014) aimed to accurately identify transcription start sites (TSSs). The experimental evidence for the TSS locations is crucial for reliable operon annotation, as well as for the detection of promoters and translation initiation sites (TISs) (Creecy and Conway 2015).

\footnotetext{
бoint first authors

Corresponding author: borodovsky@gatech.edu

Article published online before print. Article, supplemental material, and publication date are at http://www.genome.org/cgi/doi/10.1101/gr.230615.117.
}

The sequence downstream from TIS is supposed to code for interactions between mRNA and the translation machinery. In prokaryotes, translation initiation is assumed to be facilitated by the base-pairing between the $3^{\prime}$ tail of the $16 \mathrm{~S}$ rRNA of the $30 \mathrm{~S}$ ribosomal subunit and the ribosome binding site (RBS) located in the 5' UTR of the mRNA. The pioneer work of Shine and Dalgarno on Escherichia coli described a specific RBS consensus observed later in many prokaryotic genomes (Shine and Dalgarno 1974; Barrick et al. 1994). Still, with the accumulation of genomic data, exceptions started to multiply. For instance, the $5^{\prime}$ UTR may be completely absent in the case of leaderless transcription, first discovered in the archaea Pyrobaculum aerophilum (Slupska et al. 2001).

Recent studies of prokaryotic transcriptomes, including dRNA-seq applications, detected instances of leaderless transcription not only in archaea but in bacteria as well (Cortes et al. 2013). Importantly, the fraction of genes with leaderless transcription was observed to vary significantly among species. It was low ( $<8 \%$ among all operons) in some bacteria, such as Helicobacter $p y-$ lori (Sharma et al. 2010), Bacillus subtilis (Nicolas et al. 2012), Salmonella enterica (Kroger et al. 2013), Bacillus licheniformis (Wiegand et al. 2013), Campylobacter jejuni (Dugar et al. 2013),

(C) 2018 Lomsadze et al. This article is distributed exclusively by Cold Spring Harbor Laboratory Press for the first six months after the full-issue publication date (see http://genome.cshlp.org/site/misc/terms.xhtml). After six months, it is available under a Creative Commons License (Attribution-NonCommercial 4.0 International), as described at http://creativecommons.org/licenses/by$\mathrm{nc} / 4.0 /$. 
Propionibacterium acnes (Pfeifer-Sancar et al. 2013), Shewanella oneidensis (Shao et al. 2014), and Escherichia coli (Thomason et al. 2015). It was also low $(<15 \%)$ in some archaea, e.g., in Methanosarcina mazei (Jager et al. 2009), Pyrococcus abyssi (Toffano-Nioche et al. 2013), Thermococcus kodakarensis (Jager et al. 2014), Methanolobus psychrophilus (Li et al. 2015), and Thermococcus onnurineus (Cho et al. 2017). However, a higher frequency $(>25 \%)$ of leaderless transcription was observed in other bacteria, e.g., Mycobacterium tuberculosis (Cortes et al. 2013), Corynebacterium glutamicum (Pfeifer-Sancar et al. 2013), Deinococcus deserti (de Groot et al. 2014), Streptomyces coelicolor (Romero et al. 2014), Mycobacterium smegmatis (Shell et al. 2015), and an even larger frequency (>60\%) was seen in various archaeal species, e.g., Halobacterium salinarum (Koide et al. 2009), Sulfolobus solfataricus (Wurtzel et al. 2010), and Haloferax volcanii (Babski et al. 2016). Accordingly, the diversity of regulatory sequence patterns that appear near gene starts motivated the effort to build multiple models necessary for more accurate gene-start prediction.

Current prokaryotic gene-finding tools, GeneMarkS, Glimmer3, and Prodigal are known for a sufficiently high accuracy in predicting protein-coding ORFs. Indeed, on average these tools are able to find more than $97 \%$ of genes in a verified test set in terms of correct prediction of the gene $3^{\prime}$ ends (Besemer et al. 2001; Delcher et al. 2007; Hyatt et al. 2010). Furthermore, the accuracy of pinpointing gene starts is, on average, 90\% (Hyatt et al. 2010). We observed that most of the genes that escaped detection altogether (false negatives) belonged primarily to the atypical category, i.e., genes with sequence patterns not matching the species-specific model trained on the bulk of the genome (Borodovsky et al. 1995).

Given the high accuracy of the current tools, the task to improve prokaryotic gene finding is challenging. That said, we describe below a method that not only improves the accuracy of gene prediction across the wide range of prokaryotic genomes but also identifies genome-wide features of transcription and translation mechanisms.

\section{Methods}

\section{Gene and genome modeling}

\section{Model of a protein-coding sequence}

GeneMarkS-2 uses a rather complex model of a gene, a building block of the model of a prokaryotic genome (Fig. 1). The majority of protein-coding regions in prokaryotic genomes are known to carry species-specific oligonucleotide (e.g., codon) usage patterns (Fickett and Tung 1992). To that effect, GeneMarkS-2 learns this pattern and estimates the parameters of the typical model of protein-coding regions, a three-periodic Markov chain (Borodovsky et al. 1986a,b), by iterative self-training on the whole genome, a procedure similar in general to the one introduced in GeneMarkS (Besemer et al. 2001).

Still, the oligonucleotide composition of some genes may deviate from the genome-wide mainstream. For that reason, GeneMarkS introduced an additional atypical gene model along- side the typical model (Besemer et al. 2001). Instead of a single atypical model, GeneMarkS-2 uses two large sets of atypical models: 41 bacterial and 41 archaeal. The precomputed parameters of the atypical models, covering the GC content range from $30 \%$ to $70 \%$, were estimated by a method utilized earlier in the metagenome gene finder (Zhu et al. 2010); these parameters are not re-estimated in GeneMarkS-2 iterations. Each atypical model has a GC content index indicating a narrow (1\%) GC bin that it represents. The GC of the candidate ORF is used to identify which of the atypical models should be deployed for that ORF analysis. As such, only a subset of the atypical models is used in a GeneMarkS-2 run on any given genome.

This multimodel approach could be interpreted in the following way. Disregarding for a moment the linear connectivity of genes in a given genome, we can think of this set of 'disjoint' genes as an instance of a small 'metagenome.' The approach developed earlier for metagenome analysis (Zhu et al. 2010) employed a variety of models for the analysis of sequence fragments with varying GC contents; we use this library as the set of atypical models for GeneMarkS-2. Furthermore, the typical genes, making the majority of this disjoint gene set, could be effectively clustered and processed together to determine parameters for the typical model. With this set of models at hand, a given ORF is predicted as a gene by the best fitting model, i.e., the model (the typical or the GC-matching atypical) that yields the highest score.

\section{Model of a sequence around the gene start}

Sequence patterns near gene starts are species-specific; nevertheless, we identified groups of genomes with similar patterns that arguably were selected in evolution due to the common features of translation and transcription mechanisms. We have introduced four distinct categories/groups of the pattern models (A through D). We also observed that some genomes did not fall into one of these four groups; as such, we created the fifth category/group $\mathrm{X}$ to which all such genomes were assigned. In general, category $\mathrm{X}$ either indicates very weak regulatory signals (hard to detect and classify) or possibly signals related to a new and yet poorly characterized translation initiation mechanism.

The first, group A, represents genomes with an observed dominance of RBS sites having the Shine-Dalgarno (SD) consensus; the usage of leaderless transcription was negligible or nonexistent in such genomes. Genomes of the second category, group B, carry RBS sites with a "non-Shine-Dalgarno" (non-SD) consensus. Next, there are group $\mathrm{C}$ and group $\mathrm{D}$ which represent bacterial

\section{Genome Research}

www.genome.org 
and archaeal genomes, respectively, characterized by a significant presence of leaderless transcription. Importantly, in group $\mathrm{C}$ genomes, the bacterial promoter signal (Pribnow box) is located at $\sim 10$ nt distance from the start of the first gene in operon (transcribed in the leaderless fashion). In group $\mathrm{D}$, the archaeal promoter box is situated at $\sim 26 \mathrm{nt}$ distance from the starts of genes with leaderless transcription. Furthermore, other genes (i.e., internal genes in operons or first-genes-in-operons with leadered transcription) in genomes of groups $\mathrm{C}$ and $\mathrm{D}$ are likely to use RBS sites; we therefore use dual gene start models in these types of genomes. In group X, we saw weak, hard-to-classify regulatory signal patterns; still, some genes in genomes of group X could have an RBS signal with SD consensus.

The sites of promoter boxes as well as the non-ShineDalgarno and the Shine-Dalgarno RBS sites are separated from TISs by sequences with variable length (spacers). Therefore, the model of a regulatory signal should include the model of the site as well as the length distribution of the spacer (Fig. 1). We also explicitly modeled the triplet upstream of the start codon as a 3nt-long upstream signature. In addition, we also used a downstream signature model (Fig. 1) that captured the patterns within the short sequence (with length up to $12 \mathrm{nt}$ ) located immediately downstream from the start codon (Shmatkov et al. 1999).

For genomes in group X, we used a combination of the site model for RBSs (if a very weak but nevertheless statistically significant RBS model can be found) and a 20-nt-long extended upstream signature (Fig. 1) in an attempt to capture some patterns not represented by the models developed for groups A through D. A brief outline of groups A-D and $\mathrm{X}$ is given in Table 1 .

\section{Unsupervised training}

The unsupervised training algorithm makes several two-step iterations (Fig. 2). Each iteration results in (1) genome segmentation into protein-coding (CDS) and noncoding regions (gene prediction), and (2) model parameters re-estimation (Besemer et al. 2001).

\section{The first iteration}

\section{Prediction step}

The log-odds space Viterbi algorithm (see Supplemental Materials) computes the maximum likely sequence of hidden states (Fig. 1) along the genome, the sequence delivering the largest log-odds score. At the first step, the algorithm only uses heuristic (atypical) models. When the algorithm runs on a particular segment of the genomic sequence, it utilizes only two of the 82 possible atypical models: specifically, the two, bacterial and archaeal, whose GC index matches the local sequence's GC composition. In the first iteration, the algorithm does not account for sequence patterns near gene starts since no gene-start model has been derived yet.

\section{Estimation step}

After the first run of the Viterbi algorithm, all genomic segments predicted and labeled as 'protein-coding' are collected into a training set to estimate the parameters of the 'typical' model. To reduce the chance of including noncoding ORFs into the training set, predicted genes shorter than $300 \mathrm{nt}$ and those predicted as incomplete CDS are not used in training. From a thus complied sequence set, the typical model, a fifth order three-periodic Markov model, is derived (Borodovsky and McIninch 1993). Similarly, the genomic segments labeled as 'noncoding' are used to estimate parameters for the noncoding model, structured as a second order uniform Markov chain.

\section{The second iteration}

\section{Prediction step}

The Viterbi algorithm is executed to produce the updated genome segmentation. Note that at this iteration we use the newly derived typical model along with all the atypical models. In the estimation step, the repredicted genome segmentation is used to re-estimate parameters of the typical model. At this time, having information on initially predicted gene starts, the algorithm selects sequences situated around the gene starts and derives models of the patterns encoding transcription and/or translation regulation (Supplemental Fig. S1).

\section{Building the models of sequences around gene starts}

In general, the sites near the first genes in operons (FGIOs) regulate transcription and translation (promoters and RBS sites), while the sites located near the interior genes in operons (IGIOs) regulate translation (RBS sites only). Given a predicted genome segmentation into protein-coding and noncoding regions, we identify FGIOs by the following rule. A gene is an FGIO if its upstream gene neighbor is located in the same strand at a distance larger than $25 \mathrm{nt}$ or in the complementary strand (at any distance). The set of FGIOs is updated at each iteration. The rationale for choosing the 25-nt threshold was as follows. Intuitively, larger values offer a more conservative selection of operons and FGIOs, since distances between operons tend to be larger than distances between genes in operons. The operons annotated in the $E$. coli genome (Gama-Castro et al. 2016) were used to assess the effect of the threshold value on the accuracy of operon delineation. The 25 -nt cut-off identified $98 \%$ of the annotated FGIOs correctly, while making $\sim 8 \%$ false positive predictions. Further, although a 40-nt threshold could offer a slightly better balance between true and false operon predictions (Supplemental Fig. S2), the experiments showed that the 40-nt threshold produced an equal (or even slightly worse) gene and gene-start prediction accuracy.

Table 1. Features of the regulatory site models used for genomes of Groups A-D and X

\begin{tabular}{|c|c|c|c|c|c|}
\hline Groups & Transcription features \& domain & Representative species & RBS consensus & $\begin{array}{l}\text { Promoter box } \\
\text { localization }\end{array}$ & $\begin{array}{l}\text { Extended upstream } \\
\text { signature }\end{array}$ \\
\hline A & Leadered with predominantly SD RBS & Escherichia coli & SD & - & - \\
\hline B & Leadered with predominantly non-SD RBS & Bacteroides ovatus & non-SD & - & - \\
\hline C & Leaderless \& bacteria & Mycobacterium tuberculosis & SD & 10 & - \\
\hline D & Leaderless \& archaea & Holobacterium salinarum & SD & 26 & - \\
\hline $\mathrm{x}$ & Unclassified & Synechocystis & SD & - & $20 \mathrm{nt}$ \\
\hline
\end{tabular}

A dash indicates that a particular model was not used; 26 and 10 indicate average nt distance between the promoter Pribnow box and the position of translation start. 


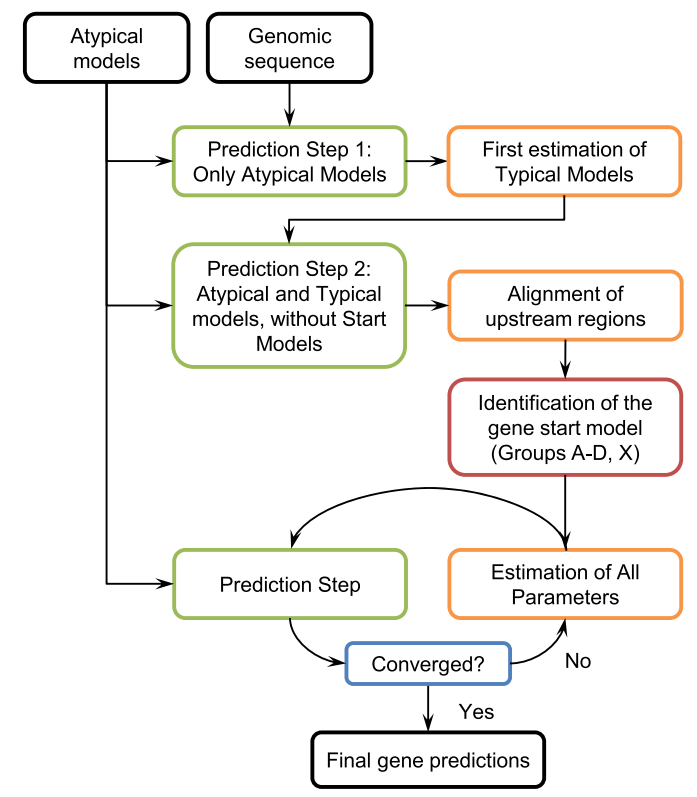

Figure 2. Principal workflow of the unsupervised training.

The two generic components of a regulatory site model, the nucleotide frequency matrix and the spacer length distribution, are then derived by running the modified Gibbs sampler algorithm GibbsL (described below). In some situations, it is useful to assess the "validity" of the motif by examining the preferred localizations of the motif instances. To do this, we define the localization distance as the mode of the spacer length distribution, i.e., the most frequent distance between the motif and the gene start. If the frequency at the mode is larger than a threshold value $\mathrm{Q} \%$, then the motif is considered to be localized.

The full details of the derivations of the models (for groups A-D and X) illustrated by the 'logos' of positional frequency patterns of the motifs and the associated spacer length distributions (Supplemental Figs. S3-S7) are given in the Supplemental Materials.

\section{The third iteration and on}

From now on, the type of the model of the gene start (and, therefore, the group to which the genome is supposed to "belong") does not change. However, the model parameters are updated in iterations with respect to the type-specific rules. At each prediction step, the dual motif models of the RBS and promoter box models (defined for both groups $\mathrm{C}$ and D) compete in the Viterbi algorithm computations. Similarly, the RBS and extended upstream signature models compete when the group X genome is processed. The typical gene model is updated at each iteration (Fig. 2) using the latest version of the genome segmentation. GeneMarkS-2 continues the prediction/estimation iterations until the convergence condition is fulfilled (a 99\% identity in gene starts between consecutive iterations). Alternatively, the algorithm stops if the maximum number of iterations is reached ( 10 by default). All the sequence segments labeled as coding regions in the final iteration are reported as predicted genes.

\section{A motif finder that accounts for the signal localization pattern}

The MCMC motif finder Gibbs3 (Thompson et al. 2003) was designed to learn a probabilistic model of an a priori unknown motif present in a set of sequences. Gibbs3 was used for the RBS model delineation in GeneMarkS with reasonable accuracy (Besemer et al. 2001).

It was observed that sequences separating regulatory motifs from gene starts (spacers) have some preferred (more frequent) lengths, presumably facilitating molecular interactions involved in translation initiation. However, the Gibbs 3 algorithm treats motif instances with spacer lengths that are too long or too short in the same way as the motif instances having a more optimal spacer length.

Therefore, we implemented a modified Gibbs sampler type algorithm, called GibbsL (see Supplemental Materials). We explicitly included the spacer length into the objective function in order to penalize motifs that appear at distances that vary significantly from the optimal locations. At a given iteration of GeneMarkS-2, GibbsL runs a fixed number, $N$, of its own iterations to derive a full model for a regulatory site (default $N=60$ ). Furthermore, the instances of GibbsL runs are repeated $M$ times to mitigate the effect of random initializations (default $M=30$ ); then, the result with the highest objective function value is selected.

\section{Test set—genes supported by proteomic studies}

Mass-spectrometry-determined peptides were obtained in studies of a number of prokaryotic species at the Pacific Northwest National Laboratory (Venter et al. 2011). From all the available genomes, we selected 54 , each with more than 250 proteomics-validated ORFs (supported by at least two matching peptides). The peptide-supported ORFs (psORFs) annotated in the 54 genomes (Supplemental Table S1) were used in the assessment of false negative and false positive rates of gene prediction.

\section{Test set—COG annotated genes}

We used 145 genomes (115 bacteria and 30 archaea covering 22 bacterial and archaeal phyla, listed in Supplemental Table S2) that varied in size, type of genetic code, and GC content. Among the genes annotated in these genomes, we selected genes with the COG characterization; therefore, the orthologous relationships of their protein products with proteins from other species were earlier established within the Clusters of Orthologous Groups (Tatusov et al. 1997, 2003; Galperin et al. 2015). The COG annotation serves as robust evidence of the functional role of a gene, thus this gene set is unlikely to include random ORFs. Since 36 out of 145 genomes belonged to the set of 54 genomes with 'proteomics'-confirmed genes, we removed the redundancy in the actual tests (see below).

\section{Test sets with simulated genome-specific noncoding regions}

We used annotated intergenic regions of each of 145 genomes (Supplemental Table S2) to build models of noncoding sequence (zero-order Markov chains). Notably, such a simple type of model of the noncoding region was not used in any of the four gene finders. We employed these models to generate species-specific random noncoding sequences and used them as follows. Firstly, in each genome out of the 145 mentioned above (excluding Mycobacterium leprae, known for large numbers of pseudogenes), we replaced intergenic regions by simulated noncoding sequences of the same length as in the original genome (Set 1). Secondly, we employed the models to generate 145 sequences of length 100,000 nt (Set 2). Thirdly, we added 100,000 nt of simulated noncoding sequence to the $3^{\prime}$ end of each genome (Set 3).

\section{Test sets of genes with experimentally verified starts}

$\mathrm{N}$-terminal protein sequencing is a standard technique to validate sites of translation initiation (protein $\mathrm{N}$-terminals and gene starts).

\section{Genome Research}

www.genome.org 
Relatively large sets of genes with validated starts were known for the bacteria Synechocystis sp. (Sazuka et al. 1999), E. coli (Rudd 2000; Zhou and Rudd 2013), M. tuberculosis (Lew et al. 2011), and $D$. deserti (de Groot et al. 2014) and the archaea Aeropyrum pernix (Yamazaki et al. 2006), H. salinarum, and Natronomonas pharaonis (Aivaliotis et al. 2007).

\section{Set of representative prokaryotic genomes}

The prokaryotic genome collection of NCBI includes a description of 5007 species as 'representatives' of the whole database of more than 100,000 genomes (Tatusova et al. 2014). These include 238 archaeal and 4769 bacterial species to cover all the genera, while leaving out the majority of species of the respective genera along with most of their strains.

\section{Results}

Error rates in prediction of protein-coding genes (all but the $5^{\prime}$ end)

Gene predictions made by GeneMarkS, Glimmer3, Prodigal, and GeneMarkS-2, run with default settings, were compared with (1) annotation of the MS or 'proteomics' validated genes, and (2) the COGs-validated genes. In the 54 genomes (Supplemental Table S2), there were $\sim 89,500$ proteomics-supported genes (psORFs) (Table 2); in the 145 genomes (Supplemental Table S3), there were $\sim 341,486$ genes in total, 287,237 of which did not overlap with the proteomics-validated genes (Tables 2, 3).

In the set of 54 genomes, we observed that GeneMarkS-2 missed 181 psORFs out of 89,466 , the least number of false negative errors made by the tested tools (Table 2). At the same time, GeneMarkS-2 made the least number of false positive predictions, 114. A predicted gene was judged as false if more than $30 \%$ of its length overlapped with a psORF located in one of five other frames.

The comparison of the predictions with the COG-validated genes demonstrated higher accuracy of GeneMarkS-2 as well. The new tool missed the lowest number of COG genes, 1147, fol-

Table 2. Statistics of false negative (panel $A$ ) and false positive gene predictions (panel $B$ ) observed in tests on 54 genomes containing proteomic-validated genes and on $\mathbf{1 4 5}$ genomes with genes validated by orthologs in COGs

A

\begin{tabular}{lcc}
\hline Algorithm & $\begin{array}{c}\text { Missed MS } \\
\text { confirmed genes } \\
\text { (from 89,466) }\end{array}$ & $\begin{array}{c}\text { Missed COG genes } \\
\text { (not MS) } \\
\text { (from 287,237) }\end{array}$ \\
\hline GeneMarkS & 376 & 1467 \\
Glimmer3 & 496 & 1990 \\
Prodigal & 217 & 1389 \\
GeneMarkS-2 & 181 & 1147 \\
\hline B & &
\end{tabular}

B

\begin{tabular}{lcc}
\hline Algorithm & $\begin{array}{c}\text { False predictions } \\
\text { overlapping } \\
\text { MS-confirmed genes }\end{array}$ & $\begin{array}{c}\text { False predictions } \\
\text { overlapping } \\
\text { COG genes (not MS) }\end{array}$ \\
\hline GeneMarkS & 352 & 2046 \\
Glimmer3 & 921 & 6435 \\
Prodigal & 211 & 1339 \\
GeneMarkS-2 & 114 & 932 \\
\hline
\end{tabular}

lowed by Prodigal with 1389. Notably, the rate of missed COG genes by any single gene finder was $<1 \%$ (Table 2 ). Counting false positives (identified also as ones with prohibitively long overlaps with verified genes) has shown that GeneMarkS-2 made 932 false predictions, a significantly smaller number than the ones made by the other gene finders (Table 2). Note that the COG-validated genes identical to the 'proteomics' supported genes were excluded from the second test.

We specifically looked into the distributon of the two types of errors with respect to the gene length (Table 3). Among all COGannotated genes, Glimmer3 missed the least number of short genes (in the 90- to 150-nt range) in comparison with the other tools (Table 3A). In the next bin, 150-300 nt, GeneMarkS did show the best result. We observed that GeneMarkS- 2 missed the least numbers of COG genes with length $>300 \mathrm{nt}$ and made the least total count (Table 3A). Its performance was the least dependent on genome GC content (Supplemental Fig. S8).

Since it turned out that the false positives identified by their too long overlaps with validated genes (confirmed by proteomics or by COG annotation) occurred in rather small numbers (Table 2), we attempted to offer more substantial statistics by adding tests on sets of synthetic sequences.

\section{False positive predictions in synthetic sequences}

We estimated the false positive rates on the three sets described above. First, we ran the four gene finders on Set 1, the 144 constructs where the intergenic sequences were replaced by the same length synthetic noncoding sequences, while the annotated genes remained in place. The predicted genes with the $3^{\prime}$ end not matching annotation were considered as false positives (Table 3B). Notably, the number of false negatives in these experiments was observed to be of the same order as in the runs of the gene finders on the original genomes, i.e., $\sim 1 \%$ of the number of annotated genes. Second, we ran the four gene-finding tools trained on 145 complete genomes on Set 2, the 145 sequences of length $100,000 \mathrm{nt}$, with the experiment repeated 10 times (Supplemental Table S5). The first and the second types of experiments had the advantage of keeping the trained parameters consistent with the nonperturbed features of the genome. However, the gene finders were not adapted to the simulated noncoding sequences. Therefore, we ran the gene finders in full cycle, training and prediction, on Set 3 where predictions made in the 100,000-nt extended artificial portion of each genome were counted as false positives (Supplemental Table S6).

The results of all the three types of tests described above were favorable for GeneMarkS-2. Further analysis demonstrated that reduction of false positives in GeneMarkS-2 in comparison with GeneMarkS was mainly due to the improvement of the parameterization of the atypical models. Glimmer3 has made frequent false positives predictions of the short length $(<150 \mathrm{nt})$. This outcome is, arguably, the cost of its higher sensitivity in this length range than other gene finders (Table 2A).

Prodigal generated false positive predictions of rather long length, as the algorithm settings give high weights to longer ORFs. Subsequently, it leads to an increase of false positives in genomic sequences with high GC content (Supplemental Fig. S8A), where longer ORFs appear more frequently than in low GC genomes.

In all the five approaches to the assessment of false positive rates (two in natural sequences and three in artificial ones), we saw that GeneMarkS-2 demonstrated the best performance. 
Table 3. Statistics of false negative (panel $A$ ) and false positive (panel $B$ ) gene predictions

\begin{tabular}{|c|c|c|c|c|c|c|c|}
\hline A & Bins (nt) & $<150$ & $150-300$ & $300-600$ & $600-900$ & $>900$ & Total \\
\hline \multirow[t]{2}{*}{ Algorithm } & COG genes & 362 & 13,985 & 65,948 & 83,745 & 177,446 & 341,486 \\
\hline & & \multicolumn{6}{|c|}{ Missed annotated genes (FN) } \\
\hline GeneMarkS & & 136 & 494 & 434 & 192 & 296 & 1552 \\
\hline Glimmer3 & & 66 & 678 & 1170 & 341 & 323 & 2578 \\
\hline Prodigal & & 161 & 639 & 417 & 92 & 78 & 1387 \\
\hline GeneMarkS-2 & & 132 & 596 & 370 & 76 & 69 & 1243 \\
\hline B & Bins (nt) & $<150$ & $150-300$ & $300-600$ & $600-900$ & $>900$ & Total \\
\hline Algorithm & & \multicolumn{6}{|c|}{ False positives (FP) in simulated sequence } \\
\hline GeneMarkS & & 3366 & 5113 & 1230 & 177 & 94 & 9980 \\
\hline Glimmer3 & & 17,446 & 5044 & 1299 & 228 & 136 & 24,153 \\
\hline Prodigal & & 4525 & 5321 & 1453 & 419 & 135 & 11,853 \\
\hline GeneMarkS-2 & & 792 & 1541 & 601 & 137 & 77 & 3148 \\
\hline
\end{tabular}

Panel A: Counts of genes missed by a particular tool (false negatives) among 341,486 COG genes annotated in 145 genomes. The counts are given in five length bins. Panel $B$ : Counts of false positive predictions made in 144 simulated genomic sequences made from 144 original genomes where annotated intergenic regions were replaced by artificial noncoding sequence (see text). The numbers of false predictions were sorted by length in the same way as in Panel $A$. Bold font designates the minimal number of observed errors in each column (for each panel separately).

Notably, it yielded the lowest numbers of false positives in all the length intervals.

\section{Accuracy of gene start prediction}

As was stated above, we collected data on genes with experimentally validated translation initiation starts. Currently available collections were limited to the species of A. pernix, D. deserti, E. coli, H. salinarum, M. tuberculosis, N. pharaonis, and Synechocystis sp. To assess the accuracy of gene start prediction, we compared positions of predicted and annotated starts of these verified genes.

The observed error rate of GeneMarkS- 2 was $4.4 \%$, followed by Prodigal at $6.1 \%$, GeneMarkS at $10.2 \%$ and finally, Glimmer3 at $13.2 \%$. Therefore, GeneMarkS-2 made the largest number of correct predictions among the four gene finders (Table 4).

In comparison with GeneMarkS, the improved start prediction accuracy of GeneMarkS-2 was due to a more flexible modeling of the regulatory signals near gene starts. For instance, in the group C genome of M. tuberculosis, GeneMarkS did not find a sufficiently strong RBS motif (Fig. 3A). GeneMarkS-2, on the other hand, predicted that $40 \%$ of operons are likely to be transcribed in the leaderless fashion, with the promoter Pribnow box located at a 6- to 8-nt distance from the gene starts (Fig. 3B). In the remaining $\sim 60 \%$ of operons, predicted RBS sites were separated from the gene starts by the same 6- to 8-nt distance (Fig. 3C). Therefore, the mixture of patterns, promoters, and RBS sites, localized on the same distance from the gene start, was the reason for GeneMarkS to fail to converge to an informative motif model.

In another example, for the majority of the first genes in operons in the group D archaea H. salinarum, GeneMarkS-2 identified the promoter Pribnow box located at 22-24 nt from the gene starts (Fig. 3E), at the distance characteristic for leaderless transcription in archaeal genomes. For the remaining FGIOs as well as for IGIOs, GeneMarkS-2 identified the RBS sites at a 6- to 8-nt distance upstream of gene starts (Fig. 3F). GeneMarkS, which assumes a single type of motif for all FGIOs, could only derive a Pribnow boxlike site motif with a weaker localization (Fig. 3D).

On a general note, the performance of GibbsL in detecting RBS motifs with the Shine-Dalgarno consensus in genomes with low and mid GC content was observed to be similar to the performance of Gibbs3 (though GibbsL tends to have higher localization peaks). However, in genomes with high GC content, GibbsL derived motifs with a higher information content and a more compact localization (Supplemental Figs. S9-S11). We also observed that GibbsL was more robust than Gibbs3 upon increasing the length of sequences (i.e., the selected gene upstream regions) supposed to contain common motifs (Supplemental Figs. S9, S10).

Table 4. Numbers of correctly predicted gene starts verified by $\mathrm{N}$-terminal protein sequencing

\begin{tabular}{|c|c|c|c|c|c|c|}
\hline Species & Gene-start model type & \# of verified gene starts & GeneMarkS & Glimmer3 & Prodigal & GeneMarkS-2 \\
\hline A. pernix ${ }^{\mathrm{a}}$ & A & 130 & 125 & 119 & 127 & 126 \\
\hline D. deserti & C & 384 & 315 & 314 & 334 & 369 \\
\hline E. coli & A & 769 & 725 & 714 & 751 & 740 \\
\hline H. salinarum ${ }^{\mathrm{a}}$ & D & 530 & 502 & 454 & 514 & 523 \\
\hline M. tuberculosis & C & 701 & 572 & 572 & 620 & 635 \\
\hline N. pharaonis ${ }^{\mathrm{a}}$ & $\mathrm{D}$ & 315 & 309 & 288 & 309 & 312 \\
\hline \multirow{2}{*}{ Synechocystis } & $x$ & 96 & 81 & 79 & 92 & 92 \\
\hline & Total & 2925 & 2629 & 2540 & 2747 & 2797 \\
\hline
\end{tabular}

Bold font designates the maximum number of correct start predictions for each species as well as in total. ${ }^{\text {a Archaea. }}$ 


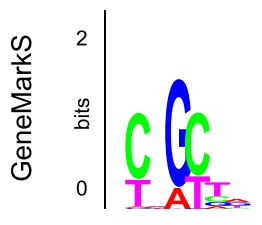

M. tuberculosis
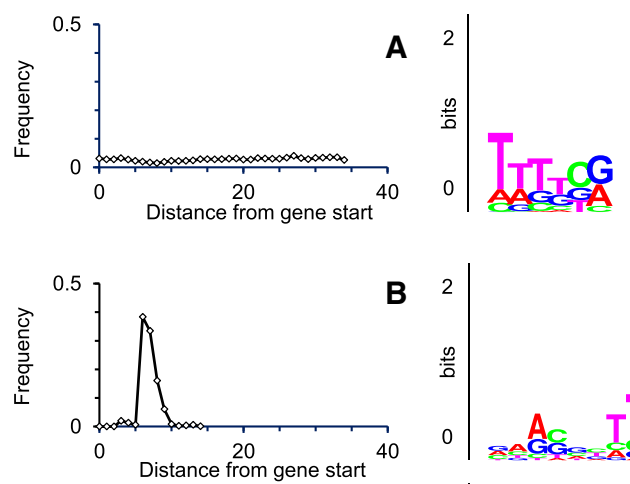

C

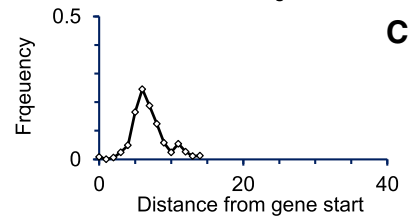

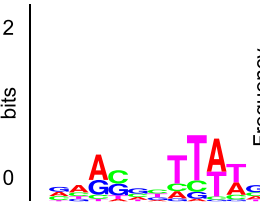

H. salinarum
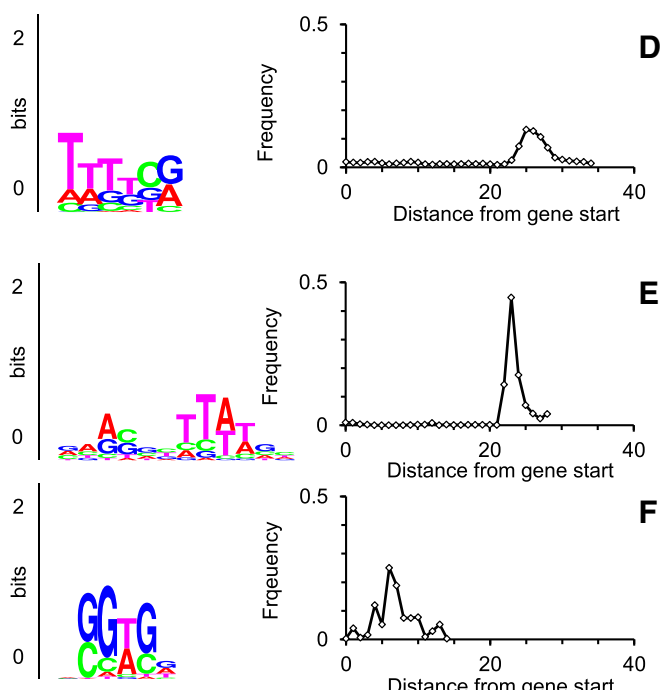

D
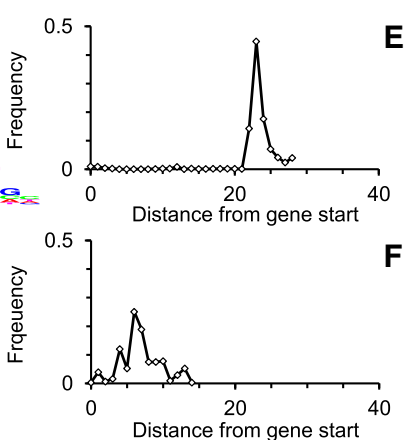

Figure 3. Motif logos and spacer length distributions for genomes of $M$. tuberculosis (Group C) and H. salinarum (Group D). In M. tuberculosis, the 'mixed' motif found by GeneMarkS has no preferred localization (panel $A$ ) in the upstream regions of the first genes in operons. To the contrary, the motif found by GeneMarkS-2 has a clear localization at -10 distance from gene starts, the distance typical for bacterial TATA box and leaderless transcription (B). In upstream regions of internal genes in operons, GeneMarkS-2 built the RBS model and the spacer length distribution (C). For $H$. salinarum, comparison of GeneMarkS-2 outcomes $(E, F)$ with ones by GeneMarkS $(D)$ shows similar improvements.

Finally, we have observed that the selection of the width of the motif in GibbsL in the range from 5 to 10 nt did not show a significant influence on gene start prediction (Supplemental Table S3). Indeed, the motifs with larger widths, i.e., $10 \mathrm{nt}$, show a minor change in comparison with the motifs with short width, e.g., $5 \mathrm{nt}$, as shown in Supplemental Figure S12.

\section{Analysis of $\sim 5000$ prokaryotic genomes with GeneMarkS-2}

Upon generating gene predictions for $\sim 5000$ representative NCBI genomes (see above), GeneMarkS-2 made an assignment of each genome into one of the five groups/categories, A-D and X. We presented the species assigned to each category in Supplemental Table S4, A-X, with names listed along the branches of five trees that follow the taxonomic order. Not surprisingly, the species from the same clades tend to belong to the same category. The distribution of the five categories at the top three taxonomical levels is shown in Figure 4.

The largest, group A, includes 2935 bacteria and 39 archaea (Supplemental Table S4A). In the species of category A, gene expression occurs predominantly via mRNAs in which $5^{\prime}$ UTRs carry detectable RBS motifs with the Shine-Dalgarno consensus. Among the category A bacteria, $61 \%$ were Gram-negative and 39\% were Gram-positive. Still, these Grampositive species in group A comprised more than half, $57 \%$, of Gram-positive bacteria in the set of $\sim 5000$ species. Furthermore, it is only Gram-positive Actinobacteria that rarely belong to group A (78 out of $859,9.1 \%)$ and mostly appear in group C; $96 \%$ of all the other Grampositive bacteria belong to group A.
The group/category B assignments were made for 495 bacteria and no archaea (Supplemental Table S4B). The characteristic feature of this category is non-SD type RBS motif. In genomes of group B, we observed the presence of the same type motif in the upstream sequences of all the genes, both initial and internal genes in operons; however, the motif did not have the Shine-Dalgarno consensus. Since this motif is present at a short distance from the translation start in the leadered mRNAs, it cannot be a promoter. Species of group B are frequent in the FCB group of bacteria (409 out of $455,89.9 \%)$, but rare in Terrabacteria (1.7\%) and Proteobacteria $(2.0 \%)$.

Group C (1028 out of 4769 bacteria) consists of bacterial species predicted to frequently have the presence of leaderless mRNAs (Supplemental Table S4C). Species of group C are frequent in Actinobacteria (773 from 859, 90.0\%) and Deinococcus-Thermus (37 out of 38, 97.4\%), but rare in Proteobacteria (104 out of 1854,
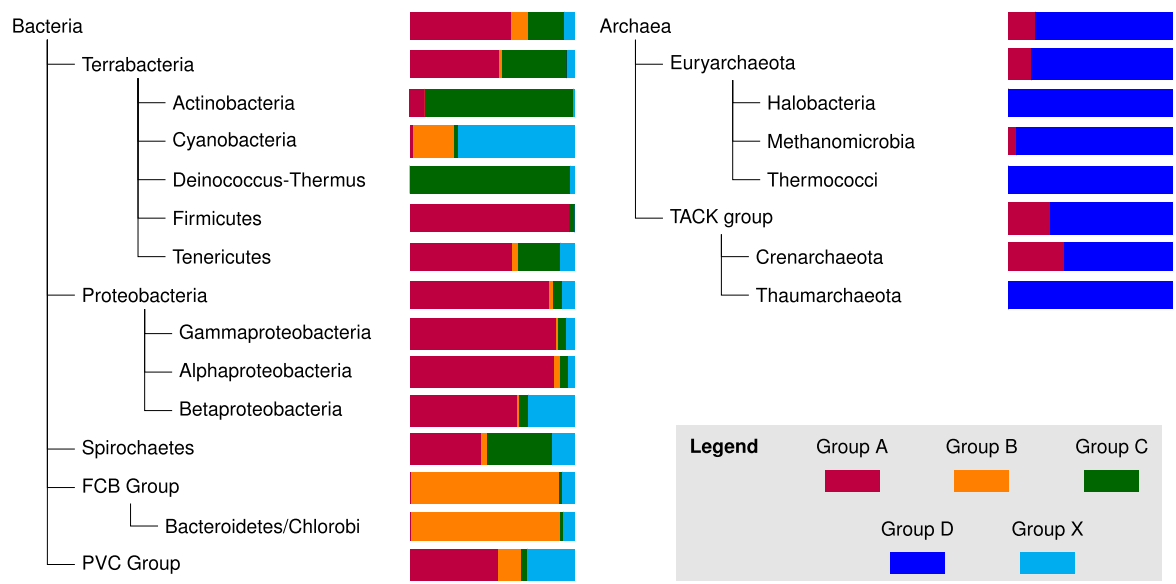

Figure 4. Color-coded scheme of the distribution of groups $A-D$ and $X$ among $\sim 5000$ representative genomes. The diagram shows the top three levels of the taxonomy trees of both archaea and bacteria. 
5.6\%) and Firmicutes (36 out of 1064, 3.4\%). A particularly high frequency of category C species was observed in Streptomycetales (129 out of $129,100 \%$ ) and in Corynebacteriales (197 out of 202, 97.5\%) including Mycobacteriaceae (56 out of 57, 98.2\%).

Next is group D that includes archaeal species with prevalence of leaderless mRNAs. A promoter box-like motif was derived in genomes of this category for leaderless FGIO, while for the remaining genes an RBS motif was determined. From the 238 archaeal genomes in our data set, 199 were assigned to group D. In particular, some taxa had most (or all) of their members belonging to this group, such as Halobacteria (74 out of 74 species, 100\%), Methanomicrobia (40 out of 42,95\%), Thermococci (21 out of 21, $100 \%)$, Thermoplasmata (11 out of 11, 100\%), Archeoglobi (7 out of 7,100\%), Thaumarchaeota (11 out of $11,100 \%)$, and Crenarchaeota (23 out of $35,65 \%$ ) (see Supplemental Table S4D). Leaderless transcription is the characteristic feature of group $\mathrm{D}$ genomes. We have inferred, however, that group A is home for a significant fraction of the taxon Crenarchaea, where $P$. aerophilum belongs. Thus, many members of Crenarchaea should have a low percentage of leaderless transcripts.

Finally, 311 bacterial species did not fit any of the above four groups (A through D) and were included into group X (Supplemental Table S4X), characterized by the (seeming) absence of pronounced regulatory signals upstream of most genes. Still, the weakness of the regulatory signal has its own commonality. Species of this group are relatively frequent in Cyanobacteria (90 out of 127, 70.9\%) and in Burkholderiales (63 out of 166, 37.9\%).

The summary list of the distribution of the $\sim 5000$ species among groups $\mathrm{A}-\mathrm{D}$ and $\mathrm{X}$ is given in Table 5 .

\section{Discussion}

\section{Gene finding accuracy evaluation}

We demonstrated in several tests that, on average, GeneMarkS-2 is a more accurate tool among the current frequently used gene finders. Particularly, GeneMarkS-2 made fewer false negative and false positive errors in predicting genes validated by mass spectrometry and COG annotation (Table 2). Also, GeneMarkS-2 made the least numbers of false positive predictions in simulated noncoding sequences (Table 3B).

The array of atypical models employed in GeneMarkS-2 improved the prediction of horizontally transferred (atypical) genes. In our observations, the deviation of GC composition of atypical genes from the genome average could be as large as $16 \%$ (e.g., the 798-nt-long E. coli gene b0546 characterized as DLP12 prophage, with GC content $36 \%$ compared to the $52 \%$ GC content of the bulk of $E$. coligenes). The GC content of atypical genes is frequently

Table 5. Distribution of archaeal and bacterial genomes among groups A-D and X

\begin{tabular}{|c|c|c|c|c|c|}
\hline & \multicolumn{2}{|c|}{$\begin{array}{l}\text { Archaeal } \\
\text { genomes }\end{array}$} & \multicolumn{2}{|c|}{$\begin{array}{l}\text { Bacterial } \\
\text { genomes }\end{array}$} & \multirow[b]{2}{*}{ Total number } \\
\hline & Number & $\%$ & Number & $\%$ & \\
\hline Group A & 39 & 16.4 & 2935 & 61.5 & 2974 \\
\hline Group B & 0 & 0.0 & 495 & 10.4 & 495 \\
\hline Group C & NA & NA & 1028 & 21.6 & 1028 \\
\hline Group D & 199 & 83.6 & NA & NA & 199 \\
\hline Group X & 0 & 0.0 & 311 & 6.5 & 311 \\
\hline Total & 238 & 100 & 4769 & 100 & 5007 \\
\hline
\end{tabular}

lower than the GC content of typical ones (Supplemental Fig. S13). The atypical genes with large GC content deviations are expected to appear more frequently in high GC genomes given the larger space for downward variation. The atypical genes may constitute a significant fraction of the whole gene complement (e.g., about $15 \%$ of genes in the E. coli genome [Borodovsky et al. 1995]). In our analysis of the $\sim 5000$ genomes, we found the distributions of the fraction of predicted atypical genes in archaea and bacteria are rather similar, with an averages close to $8 \%-9 \%$ (Fig. 5).

A comparison of the sets of COG-annotated genes missed by the three gene finders shows that genes predicted by atypical models of GeneMarkS- 2 constituted 30\% of 780 COG genes missed by Prodigal and $42 \%$ from 1605 COG genes missed by Glimmer3 (Supplemental Fig. S14). Both Prodigal and Glimmer3 employ a single model of protein-coding regions. We argue that the more accurate prediction of atypical genes requires the use of multiple models.

GeneMarkS-2 is able to characterize atypical genes as bacterial or archaeal given that the atypical models are divided into distinct bacterial and archaeal types (Zhu et al. 2010). The insights into the possible origin of likely horizontally transferred atypical genes could be particularly useful for thermophilic bacteria and mesophilic archaea.

GeneMarkS-2 made the least number of errors in gene-start predictions, Prodigal being the second-best (Table 4). Notably, Prodigal performed better on the E. coli set. However, this very set was used in the supervised training of Prodigal's gene-start prediction model (Hyatt et al. 2010). As expected, GeneMarkS-2 made more accurate predictions for genomes with noncanonical RBS sites (group C) and archaeal genomes with frequent leaderless transcription (group D). The experimental study of $D$. deserti identified 384 genes with verified translation starts, 262 of which had transcription starts annotated with dRNA-seq (de Groot et al. 2014). It was experimentally shown that 167 out of the 262 genes had leaderless transcription.

\section{Characterization of the patterns around gene starts: prediction of the extent of leaderless transcription}

Besides the gene finding per se, GeneMarkS-2, by virtue of predicting various types of regulatory motifs, provides prediction of the type of a transcript, leadered or leaderless. Screening of a large number of genomes led to conclusion that leaderless transcription is an ubiquitous feature of prokaryotes (Fig. 6).

In many archaea, $60 \%-80 \%$ of the operons are transcribed in leaderless fashion. Still, in a smaller fraction of archaeal species, this percentage is much lower, $25 \%-35 \%$, which is close to what was observed in bacteria assigned to group C (with 25\%-50\%).

We have compared predicted frequencies of leaderless transcription in several species with the frequencies determined experimentally (by dRNA-seq). The dRNA-seq method identifies positions of transcription starts with high accuracy. For Deinococcus deserti (de Groot et al. 2014), Haloferax volcanii (Babski et al. 2016), Sulfolobus solfataricus (Wurtzel et al. 2010), and M. tuberculosis (Cortes et al. 2013), the dRNA-seq experiments were conducted, and the authors determined the lengths of $5^{\prime}$ UTRs, the sequences between the transcription start and translation start provided in the public genome annotation. We selected the subsets of genes where the positions of annotated gene starts matched positions predicted by GeneMarkS-2. In archaea, $D$. deserti out of 1707 such genes, $\sim 62 \%$ were predicted leaderless by GeneMarkS2 and $62 \%$ were observed leaderless, i.e., the UTR length was

\section{Genome Research}

www.genome.org 


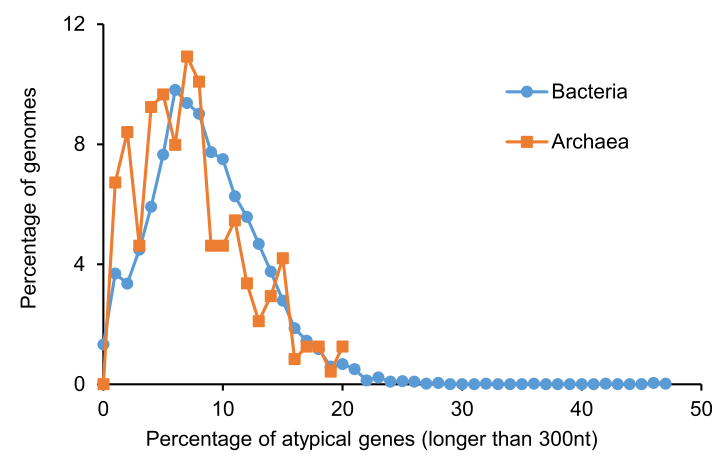

Figure 5. Distributions of the percentage of predicted atypical genes in archaeal and bacterial genomes.

$<6$ nt. In H. volcanii, the numbers were $86 \%$ and $82 \%$ (out of 1406 genes); in S. solfataricus: $78 \%$ and $76 \%$ (out of 859 genes); in bacteria, M. tuberculosis: $42 \%$ and 34\% (out of 1310 genes). Thus, the predictions were in quite good agreement for archaea and reasonably good for the single bacteria.

We observed that species where the dRNA-seq experiments found small numbers of leaderless transcripts (Sharma et al. 2010; Nicolas et al. 2012; Dugar et al. 2013; Kroger et al. 2013; PfeiferSancar et al. 2013; Wiegand et al. 2013; Shao et al. 2014; Thomason et al. 2015) were all classified as group A. Species with a large proportion of leaderless transcripts were all classified as group B (Cortes et al. 2013; Pfeifer-Sancar et al. 2013; de Groot et al. 2014; Shell et al. 2015) or group D (Koide et al. 2009; Wurtzel et al. 2010; Toffano-Nioche et al. 2013; Jager et al. 2014; Babski et al. 2016).

Experiments with Synechocystis sp. demonstrated the prevalence of leadered transcription (Mitschke et al. 2011). However, GeneMarkS-2 detected the RBS motif (with the SD consensus) only in $<15.5 \%$ of Synechocystis genes. Experiments have shown that mutating A-rich sequences situated 15-45 nt upstream of the gene starts (still within the long $5^{\prime}$ UTR) led to changes in gene expression (Mutsuda and Sugiura 2006). Nonetheless, the mechanism of the translation initiation of the majority of Synechocystis sp. genes is unknown.

In E. coli, translation initiation of the three types-SD RBSbased, non-SD RBS-based, and leaderless-is present (Shean and Gottesman 1992; Barrick et al. 1994; Resch et al. 1996). Similar observations were made for other species, and it was shown that the distribution of the number of genes controlled by each of the mechanisms could vary significantly (Gualerzi and Pon 2015). For the types rarely present in a given species, the GeneMarkS-2 training procedure is not able to make models due to the insufficient size of the training set.

\section{Regulatory motifs in the species of group B}

GeneMarkS-2 currently assigns 495 out of 4769 bacteria (and none out of 238 archaea) to group B (with non-SD RBSs). Here, we take Bacteroides ovatus as an example. Its $16 \mathrm{~S}$ rRNA features the standard 'anti-SD' pattern; however, for only 3\% of genes, the SD-matching sequences appear upstream of the gene starts. GeneMarkS-2 identified the A-rich non-SD-type motif with consistent localization at $\sim 9$ nt from the TIS (Fig. 7). The A-rich sequences appeared in upstream regions of the majority of $B$. ovatus genes. Since mutating the A-rich regions reduced gene expression levels, it was proposed that the A-rich sequences were an important part of the translation mechanism (Wegmann et al. 2013).
GeneMarkS-2 assigned 90\% of Bacteroidetes/Chlorobi species to group B (408 of 450). While not much is known about the non-SD translation mechanisms in bacteria, the clustering of the species assigned to group B within particular clades lends additional credibility to the results (Supplemental Table S4D).

Particularly, in Bacteroides (including B. ovatus), 21 out of the 23 species were assigned to group B. In their genomes, GeneMarkS2 found motifs similar in sequence and localization patterns to those of B. ovatus.

Also, 30 out of the 30 Flavobacterium species (a genus from Bacteroidetes/Chlorobi) were assigned to group B. They possessed 6-nt-long motifs, similar in sequence and localization patterns to B. ovatus (Supplemental Fig. S15 for Flavobacterium frigidarium).

Genomes of the species from these genera are closely related; nonetheless, there are differences in the derived motifs. In particular, Bacteroides tends to have a few strongly conserved A nucleotides close to the translation start, hence the peak in the spacer length distribution at $\sim 3 \mathrm{nt}$ distance from the start (Supplemental Fig. S16A). In Flavobacterium, the 'core' motif with consensus TAAAAA is more pronounced than in Bacteroides. Flavobacterium is missing strongly conserved A nucleotides near the gene start. Therefore, its 'core' motif is situated at the 3 ' end of the 15-nt window (Supplemental Fig. S16B) rather than at the $5^{\prime}$ end as in Bacteroides. The shift in the position of the 'core' motif leads to the change in the spacer length distribution which now has a peak at $\sim 7 \mathrm{nt}$. The consistency of this observation was tested for all 21 Bacteroides and 30 Flavobacteria. The 6-nt 'core' motif was not easy to detect in Prevotella (a close relative of Bacteroides) when the motif width was set to $6 \mathrm{nt}$. However, setting the motif width to $15 \mathrm{nt}$ led to the similar motif. Notably, unlike B. ovatus, other group B species may have 16S rRNA with a mutated or truncated tail (Lim et al. 2012).

In a recent publication (Nakagawa et al. 2017), species with leaderless transcription and non-SD translation initiation were included in the same class. Here, we make a distinction between these cases. The leaderless transcription excludes a possibility of RBSs appearing upstream of the first genes in operons (groups C and D). On the other hand, the RBSs with a different sequence pattern (non-SD) do appear in leadered transcripts (group B). GeneMarkS-2 is able to identify species with non-SD RBSs.

\section{Potential for further improvement}

Currently, just a small number of prokaryotic genes may escape detection by the ab initio tools, e.g., genes in which oligonucleotide composition is significantly biased, very short genes, genes

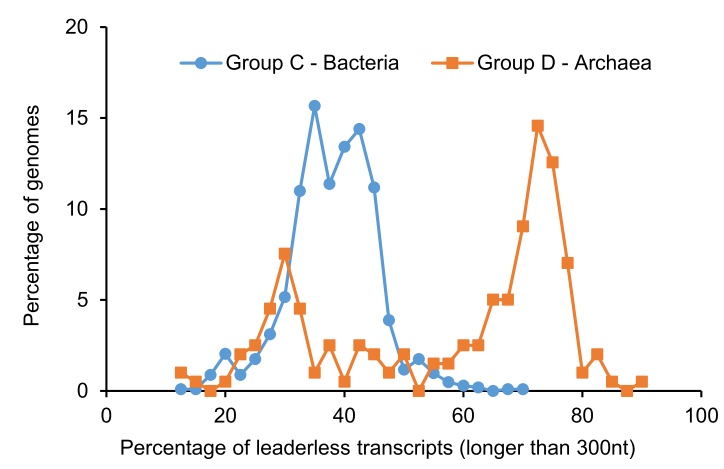

Figure 6. Distributions of the percentage of leaderless transcripts among all transcripts in bacterial Group C and archaeal Group D. 

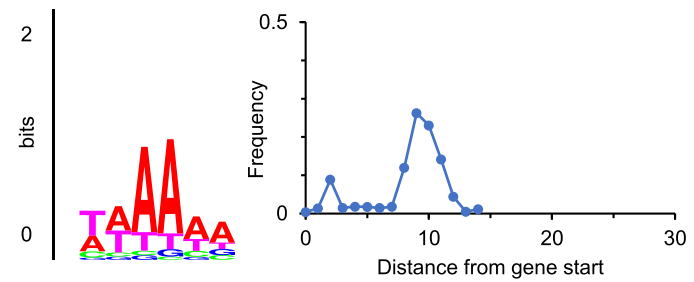

Figure 7. The motif logo and the spacer length distribution of Bacteroides ovatus, the group B genome.

containing programmed frameshifts (such as the $\operatorname{prfB}$ gene encoding a translation initiation factor [Craigen and Caskey 1986], or genes regulating some mobile elements [Sharma et al. 2011]). Furthermore, pseudogenes, especially expressed pseudogenes, could mislead gene-finding tools into generating predictions that are difficult to filter out as false positives.

An extension of GeneMarkS (known as GeneMarkS+) integrates external evidence, such as similarity to cross-species protein at a protein level or predicted RNA genes and CRISPR sequences, into ab initio gene prediction. In the NCBI genome annotation pipeline, GeneMarkS+ integrates several types of evidence into prokaryotic genome annotation (Tatusova et al. 2016). Similar extension is planned for GeneMarkS-2.

\section{Software and data availability}

The following websites can be used (1) to access the GeneMarkS-2 software: http://topaz.gatech.edu/GeneMark/genemarks2.cgi, and (2) to access data used in the course of this research project: http:// topaz.gatech.edu/GeneMark/GMS2/. Detailed descriptions of the data sets used in this paper are available either in the Supplemental Materials or at http://topaz.gatech.edu/GeneMark/GMS2/. Running time of the current version of GeneMarkS-2 is $\sim 3$ min on a genome of the size of E. coli.

\section{Acknowledgments}

We thank Susan M.E. Smith for useful comments on the manuscript. This work was supported in part by the National Institutes of Health (NIH) (HG000783 to M.B.).

\section{References}

Aivaliotis M, Gevaert K, Falb M, Tebbe A, Konstantinidis K, Bisle B, Klein C, Martens L, Staes A, Timmerman E, et al. 2007. Large-scale identification of $\mathrm{N}$-terminal peptides in the halophilic archaea Halobacterium salinarum and Natronomonas pharaonis. J Proteome Res 6: 2195-2204.

Babski J, Haas KA, Nather-Schindler D, Pfeiffer F, Forstner KU, Hammelmann M, Hilker R, Becker A, Sharma CM, Marchfelder A, et al. 2016. Genome-wide identification of transcriptional start sites in the haloarchaeon Haloferax volcanii based on differential RNA-Seq (dRNASeq). BMC Genomics 17: 629 .

Barrick D, Villanueba K, Childs J, Kalil R, Schneider TD, Lawrence CE, Gold L, Stormo GD. 1994. Quantitative analysis of ribosome binding sites in E. coli. Nucleic Acids Res 22: 1287-1295.

Besemer J, Lomsadze A, Borodovsky M. 2001. GeneMarkS: a self-training method for prediction of gene starts in microbial genomes. Implications for finding sequence motifs in regulatory regions. Nucleic Acids Res 29: 2607-2618

Borodovsky M, McIninch J. 1993. GeneMark: parallel gene recognition for both DNA strands. Compu Chem 17: 123-133.

Borodovsky M, Sprizhitskii Y, Golovanov E, Aleksandrov A. 1986a. Statistical patterns in primary structures of the functional regions of the genome of Escherichia coli. Frequency characteristics and non-uniform Markov models. Mol Biol 20: 826-840.
Borodovsky M, Sprizhitskii Y, Golovanov E, Aleksandrov A. 1986b. Statistical patterns in primary structures of the functional regions of the genome of Escherichia coli. Computer recognition of coding regions. Mol Biol 20: 1144-1150.

Borodovsky M, McIninch JD, Koonin EV, Rudd KE, Medigue C, Danchin A. 1995. Detection of new genes in a bacterial genome using Markov models for three gene classes. Nucleic Acids Res 23: 3554-3562.

Cho S, Kim MS, Jeong Y, Lee BR, Lee JH, Kang SG, Cho BK. 2017. Genomewide primary transcriptome analysis of H2-producing archaeon Thermococcus onnurineus NA1. Sci Rep 7: 43044.

Cortes T, Schubert OT, Rose G, Arnvig KB, Comas I, Aebersold R, Young DB. 2013. Genome-wide mapping of transcriptional start sites defines an extensive leaderless transcriptome in Mycobacterium tuberculosis. Cell Rep 5: $1121-1131$.

Craigen WJ, Caskey CT. 1986. Expression of peptide chain release factor 2 requires high-efficiency frameshift. Nature 322: 273-275.

Creecy JP, Conway T. 2015. Quantitative bacterial transcriptomics with RNA-seq. Curr Opin Microbiol 23: 133-140.

de Groot A, Roche D, Fernandez B, Ludanyi M, Cruveiller S, Pignol D, Vallenet D, Armengaud J, Blanchard L. 2014. RNA sequencing and proteogenomics reveal the importance of leaderless mRNAs in the radiation-tolerant bacterium Deinococcus deserti. Genome Biol Evol 6: 932-948.

Delcher AL, Bratke KA, Powers EC, Salzberg SL. 2007. Identifying bacterial genes and endosymbiont DNA with Glimmer. Bioinformatics 23: 673-679.

Dugar G, Herbig A, Forstner KU, Heidrich N, Reinhardt R, Nieselt K, Sharma CM. 2013. High-resolution transcriptome maps reveal strain-specific regulatory features of multiple Campylobacter jejuni isolates. PLoS Genet 9: e1003495

Fickett JW, Tung CS. 1992. Assessment of protein coding measures. Nucleic Acids Res 20: 6441-6450.

Galperin MY, Makarova KS, Wolf YI, Koonin EV. 2015. Expanded microbial genome coverage and improved protein family annotation in the COG database. Nucleic Acids Res 43: D261-D269.

Gama-Castro S, Salgado H, Santos-Zavaleta A, Ledezma-Tejeida D, MunizRascado L, Garcia-Sotelo JS, Alquicira-Hernandez K, Martinez-Flores I, Pannier L, Castro-Mondragon JA, et al. 2016. RegulonDB version 9.0: high-level integration of gene regulation, coexpression, motif clustering and beyond. Nucleic Acids Res 44: D133-D143.

Gualerzi CO, Pon CL. 2015. Initiation of mRNA translation in bacteria: structural and dynamic aspects. Cell Mol Life Sci 72: 4341-4367.

Hyatt D, Chen GL, Locascio PF, Land ML, Larimer FW, Hauser LJ. 2010. Prodigal: prokaryotic gene recognition and translation initiation site identification. BMC Bioinformatics 11: 119.

Jager D, Sharma CM, Thomsen J, Ehlers C, Vogel J, Schmitz RA. 2009. Deep sequencing analysis of the Methanosarcina mazei Gö1 transcriptome in response to nitrogen availability. Proc Natl Acad Sci 106: 21878-21882.

Jager D, Forstner KU, Sharma CM, Santangelo TJ, Reeve JN. 2014. Primary transcriptome map of the hyperthermophilic archaeon Thermococcus kodakarensis. BMC Genomics 15: 684.

Koide T, Reiss DJ, Bare JC, Pang WL, Facciotti MT, Schmid AK, Pan M, Marzolf B, Van PT, Lo FY, et al. 2009. Prevalence of transcription promoters within archaeal operons and coding sequences. Mol Syst Biol 5: 285.

Kroger C, Colgan A, Srikumar S, Handler K, Sivasankaran SK, Hammarlof DL, Canals R, Grissom JE, Conway T, Hokamp K, et al. 2013. An infection-relevant transcriptomic compendium for Salmonella enterica Serovar Typhimurium. Cell Host Microbe 14: 683-695.

Lew JM, Kapopoulou A, Jones LM, Cole ST. 2011. TubercuList-10 years after. Tuberculosis 91: 1-7.

Li J, Qi L, Guo Y, Yue L, Li Y, Ge W, Wu J, Shi W, Dong X. 2015. Global mapping transcriptional start sites revealed both transcriptional and posttranscriptional regulation of cold adaptation in the methanogenic archaeon Methanolobus psychrophilus. Sci Rep 5: 9209.

Lim K, Furuta Y, Kobayashi I. 2012. Large variations in bacterial ribosomal RNA genes. Mol Biol Evol 29: 2937-2948.

Locey KJ, Lennon JT. 2016. Scaling laws predict global microbial diversity. Proc Natl Acad Sci 113: 5970-5975.

Mitschke J, Georg J, Scholz I, Sharma CM, Dienst D, Bantscheff J, Voss B, Steglich C, Wilde A, Vogel J, et al. 2011. An experimentally anchored map of transcriptional start sites in the model cyanobacterium Synechocystis sp. PCC6803. Proc Natl Acad Sci 108: 2124-2129.

Mutsuda M, Sugiura M. 2006. Translation initiation of cyanobacterial $r b c S$ mRNAs requires the 38-kDa ribosomal protein S1 but not the ShineDalgarno sequence: development of a cyanobacterial in vitro translation system. J Biol Chem 281: 38314-38321.

Nakagawa S, Niimura Y, Gojobori T. 2017. Comparative genomic analysis of translation initiation mechanisms for genes lacking the Shine-Dalgarno sequence in prokaryotes. Nucleic Acids Res 45: 3922-3931.

Nicolas P, Mader U, Dervyn E, Rochat T, Leduc A, Pigeonneau N, Bidnenko E, Marchadier E, Hoebeke M, Aymerich S, et al. 2012. Condition-

\section{Genome Research}

www.genome.org 
dependent transcriptome reveals high-level regulatory architecture in Bacillus subtilis. Science 335: 1103-1106.

Pfeifer-Sancar K, Mentz A, Ruckert C, Kalinowski J. 2013. Comprehensive analysis of the Corynebacterium glutamicum transcriptome using an improved RNAseq technique. BMC Genomics 14: 888 .

Resch A, Tedin K, Grundling A, Mundlein A, Blasi U. 1996. Downstream box-anti-downstream box interactions are dispensable for translation initiation of leaderless mRNAs. EMBO J 15: 4740-4748.

Romero DA, Hasan AH, Lin YF, Kime L, Ruiz-Larrabeiti O, Urem M, Bucca G, Mamanova L, Laing EE, van Wezel GP, et al. 2014. A comparison of key aspects of gene regulation in Streptomyces coelicolor and Escherichia coli using nucleotide-resolution transcription maps produced in parallel by global and differential RNA sequencing. Mol Microbiol 94: 963-987.

Rudd KE. 2000. EcoGene: a genome sequence database for Escherichia coli K-12. Nucleic Acids Res 28: 60-64.

Sazuka T, Yamaguchi M, Ohara O. 1999. Cyano2Dbase updated: linkage of 234 protein spots to corresponding genes through N-terminal microsequencing. Electrophoresis 20: $2160-2171$.

Shao W, Price MN, Deutschbauer AM, Romine MF, Arkin AP. 2014. Conservation of transcription start sites within genes across a bacterial genus. mBio 5: e01398-01314.

Sharma CM, Vogel J. 2014. Differential RNA-seq: the approach behind and the biological insight gained. Curr Opin Microbiol 19: 97-105.

Sharma CM, Hoffmann S, Darfeuille F, Reignier J, Findeiss S, Sittka A, Chabas S, Reiche K, Hackermuller J, Reinhardt R, et al. 2010. The primary transcriptome of the major human pathogen Helicobacter pylori. Nature 464: 250-255.

Sharma V, Firth AE, Antonov I, Fayet O, Atkins JF, Borodovsky M, Baranov PV. 2011. A pilot study of bacterial genes with disrupted ORFs reveals a surprising profusion of protein sequence recoding mediated by ribosomal frameshifting and transcriptional realignment. Mol Biol Evol 28: 3195-3211.

Shean CS, Gottesman ME. 1992. Translation of the prophage $\lambda \mathrm{cl}$ transcript. Cell 70: $513-522$.

Shell SS, Wang J, Lapierre P, Mir M, Chase MR, Pyle MM, Gawande R, Ahmad R, Sarracino DA, Ioerger TR, et al. 2015. Leaderless transcripts and small proteins are common features of the Mycobacterial translational landscape. PLoS Genet 11: e1005641.

Shine J, Dalgarno L. 1974. The 3'-terminal sequence of Escherichia coli 16S ribosomal RNA: complementarity to nonsense triplets and ribosome binding sites. Proc Natl Acad Sci 71: 1342-1346.

Shmatkov AM, Melikyan AA, Chernousko FL, Borodovsky M. 1999. Finding prokaryotic genes by the 'frame-by-frame' algorithm: targeting gene starts and overlapping genes. Bioinformatics 15: 874-886.

Slupska MM, King AG, Fitz-Gibbon S, Besemer J, Borodovsky M, Miller JH. 2001. Leaderless transcripts of the crenarchaeal hyperthermophile Pyrobaculum aerophilum. J Mol Biol 309: 347-360.
Tatusov RL, Koonin EV, Lipman DJ. 1997. A genomic perspective on protein families. Science 278: 631-637.

Tatusov RL, Fedorova ND, Jackson JD, Jacobs AR, Kiryutin B, Koonin EV, Krylov DM, Mazumder R, Mekhedov SL, Nikolskaya AN, et al. 2003. The COG database: An updated version includes eukaryotes. $B M C$ Bioinformatics 4: 41

Tatusova T, Ciufo S, Fedorov B, O'Neill K, Tolstoy I. 2014. RefSeq microbial genomes database: new representation and annotation strategy. Nucleic Acids Res 42: D553-D559.

Tatusova T, DiCuccio M, Badretdin A, Chetvernin V, Nawrocki EP, Zaslavsky L, Lomsadze A, Pruitt KD, Borodovsky M, Ostell J. 2016. NCBI prokaryotic genome annotation pipeline. Nucleic Acids Res 44: 6614-6624.

Thomason MK, Bischler T, Eisenbart SK, Forstner KU, Zhang A, Herbig A, Nieselt K, Sharma CM, Storz G. 2015. Global transcriptional start site mapping using differential RNA sequencing reveals novel antisense RNAs in Escherichia coli. J Bacteriol 197: 18-28.

Thompson W, Rouchka EC, Lawrence CE. 2003. Gibbs Recursive Sampler: finding transcription factor binding sites. Nucleic Acids Res 31: 3580-3585.

Toffano-Nioche C, Ott A, Crozat E, Nguyen AN, Zytnicki M, Leclerc F, Forterre P, Bouloc P, Gautheret D. 2013. RNA at $92^{\circ} \mathrm{C}$ : the non-coding transcriptome of the hyperthermophilic archaeon Pyrococcus abyssi. RNA Biol 10: 1211-1220.

Venter E, Smith RD, Payne SH. 2011. Proteogenomic analysis of bacteria and archaea: a 46 organism case study. PLoS One 6: e27587.

Wegmann U, Horn N, Carding SR. 2013. Defining the Bacteroides ribosomal binding site. Appl Environ Microb 79: 1980-1989.

Wiegand S, Dietrich S, Hertel R, Bongaerts J, Evers S, Volland S, Daniel R, Liesegang H. 2013. RNA-Seq of Bacillus licheniformis: active regulatory RNA features expressed within a productive fermentation. $B M C$ Genomics 14: 667.

Wurtzel O, Sapra R, Chen F, Zhu Y, Simmons BA, Sorek R. 2010. A singlebase resolution map of an archaeal transcriptome. Genome Res 20: 133-141.

Yamazaki S, Yamazaki J, Nishijima K, Otsuka R, Mise M, Ishikawa H, Sasaki $\mathrm{K}$, Tago S, Isono K. 2006. Proteome analysis of an aerobic hyperthermophilic crenarchaeon, Aeropyrum pernix K1. Mol Cell Proteomics 5: $811-823$

Zhou J, Rudd KE. 2013. EcoGene 3.0. Nucleic Acids Res 41: D613-D624.

Zhu W, Lomsadze A, Borodovsky M. 2010. Ab initio gene identification in metagenomic sequences. Nucleic Acids Res 38: e132.

Received September 29, 2017; accepted in revised form May 16, 2018. 


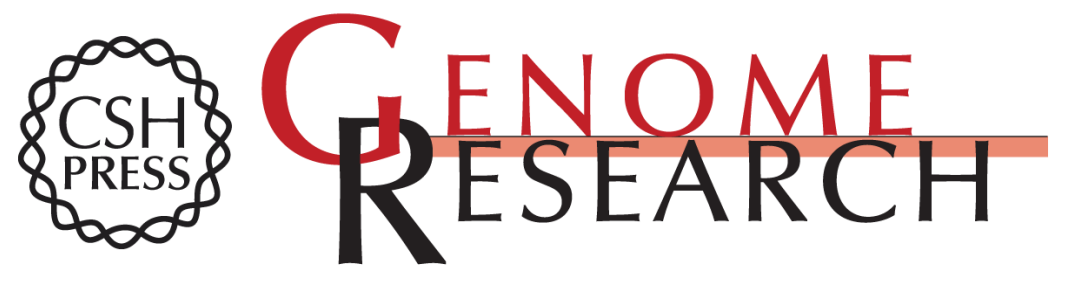

\section{Modeling leaderless transcription and atypical genes results in more accurate gene prediction in prokaryotes}

Alexandre Lomsadze, Karl Gemayel, Shiyuyun Tang, et al.

Genome Res. 2018 28: 1079-1089 originally published online May 17, 2018

Access the most recent version at doi:10.1101/gr.230615.117

Supplemental Material

References

Creative

Commons

License

Email Alerting Service
http://genome.cshlp.org/content/suppl/2018/06/14/gr.230615.117.DC1

This article cites 60 articles, 12 of which can be accessed free at: http://genome.cshlp.org/content/28/7/1079.full.html\#ref-list-1

This article is distributed exclusively by Cold Spring Harbor Laboratory Press for the first six months after the full-issue publication date (see

$\mathrm{http}: / /$ genome.cshlp.org/site/misc/terms.xhtml). After six months, it is available under a Creative Commons License (Attribution-NonCommercial 4.0 International), as described at http://creativecommons.org/licenses/by-nc/4.0/.

Receive free email alerts when new articles cite this article - sign up in the box at the top right corner of the article or click here.

\section{Affordable, Accurate Sequencing.}

To subscribe to Genome Research go to:

https://genome.cshlp.org/subscriptions 